\title{
OPEN
}

\section{Ex Situ Arterial Reconstruction During Normothermic Perfusion of the Liver}

David Nasralla, PhD, FRCS, ${ }^{1}$ Hanns Lembach, MD, ${ }^{2}$ Hynek Mergental, PhD, ${ }^{2}$ Darius Mirza, MS, FRCS, ${ }^{2}$

Peter Friend, ${ }^{3}$ Paolo Muiesan, MD, FRCS, ${ }^{2}$ and MTPR Perera, MD, FRCS ${ }^{2}$

\begin{abstract}
Background. Aberrant hepatic arterial anatomy may be seen in up to 30\% of liver grafts, and reconstruction prolongs the cold ischemic time or the arterialization times. If normothermic machine preservation (NMP) is used to preserve liver grafts, the presence of aberrant arterial anatomy poses a challenge. Dual arterial cannulation is a temporary solution to enable effective perfusion, until optimal circumstances are met for arterial reconstruction, without compromising ischemia time. To date the technical and logistical feasibility of arterial reconstruction ex situ and during NMP has not been reported. Methods. Series of 5 cases from the Consortium for Organ Preservation in Europe randomized controlled trial in which grafts with arterial anatomic variations were reconstructed while organs were perfused on NMP. Results. One donor after cardiac death and 4 donor after brain death livers with arterial anatomical variations reconstructed while on NMP were included. All patients survived transplantation, spending 1-7 d in intensive care unit and discharged home after 5-15 d. None of the cases developed early allograft dysfunction or any early technical complications. At follow-up, there were no late hepatic artery thrombosis, stenosis, or any other vascular-related complication. Four of 5 patients underwent magnetic resonance cholangiopancreatography at 6 mo with no evidence of ischemic cholangiopathy. Conclusions. The case series described above suggests that ex vivo arterial reconstruction surgery on liver grafts while on board the NMP device is feasible, safe, and effective.
\end{abstract}

(Transplantation Direct 2020;6: e596; doi: 10.1097/TXD.0000000000001040. Published online 21 August, 2020.)

\section{INTRODUCTION}

Aberrant arterial anatomy is present in approximately $30 \%$ of livers. ${ }^{1,2}$ With increasing numbers of organ procurements and transplant operations being carried out, clinicians are encountering these anomalies frequently (Figure 1). This poses a challenge during implantation as, in most recipients, it is preferable to have a single anastomosis between the donor and recipient hepatic arteries. Only very rarely is it possible to use 2 separate sources of run-in for arterial reconstructions. The options therefore in the traditional setting are to (a) perform an arterial reconstruction between the graft vessels during cold preservation before implantation (in an ice bath) or (b)

Received 20 May 2020. Revision received 18 June 2020.

Accepted 20 June 2020.

'Liver Centre, Royal Free Hospital London, London, United Kingdom.

${ }^{2}$ The Liver Unit, Queen Elizabeth Hospital Birmingham, Birmingham, United Kingdom.

${ }^{3}$ Nuffield Department of Surgical Sciences, University of Oxford, Oxford, United Kingdom.

The cases included in this article were part of the Consortium for Organ Preservation in Europe trial (ISRCTN 3973113). This study was funded by a European Commission Seventh Framework Programme (FP7) Grant (No 305934).

P.F. is cofounder, Chief Medical Officer, and consultant to OrganOX Limited and also holds shares in the company. D.M. holds minor shares in OrganOX Limited. The other authors declare no conflicts of interest. perform an additional reconstruction sequentially in situ after establishing the main arterial vascular inflow. Both of these approaches almost inevitably prolong the cold ischemic time (for ex situ reconstruction) or the period of time before arterial reperfusion (for in situ reconstruction). The nature of the arterial reconstruction varies between cases depending on the anatomical aberration.

Normothermic machine preservation (NMP) involves perfusing a liver with oxygenated blood, medications, and nutrients at normal body temperature to preserve it in a functioning, physiological state. There is accumulating evidence that NMP may improve the quality of organs at the end of preservation, while also enabling assessment of organ viability and safely extending preservation times according to logistical needs. ${ }^{3-5}$

D.N. and H.L. participated in writing of the article. H.M., D.M., P.F., and P.M. participated in intellectual content. M.T.P.R.P. participated in research design, performance of research, and writing of the article.

Correspondence: Mr Thamara Perera, Consultant Liver Transplant Surgeon, Queen Elizabeth Hospital Birmingham, Room 49E, 3rd floor Nuffield House, Birmingham, B15 2TH, United Kingdom. (Thamara.Perera@uhb.nhs.uk).

Copyright $\odot 2020$ The Author(s). Transplantation Direct. Published by Wolters Kluwer Health, Inc. This is an open-access article distributed under the terms of the Creative Commons Attribution-Non Commercial-No Derivatives License 4.0 (CCBY-NC-ND), where it is permissible to download and share the work provided it is properly cited. The work cannot be changed in any way or used commercially without permission from the journal.

ISSN: 2373-8731

DOI: 10.1097/TXD.00000000000001040 


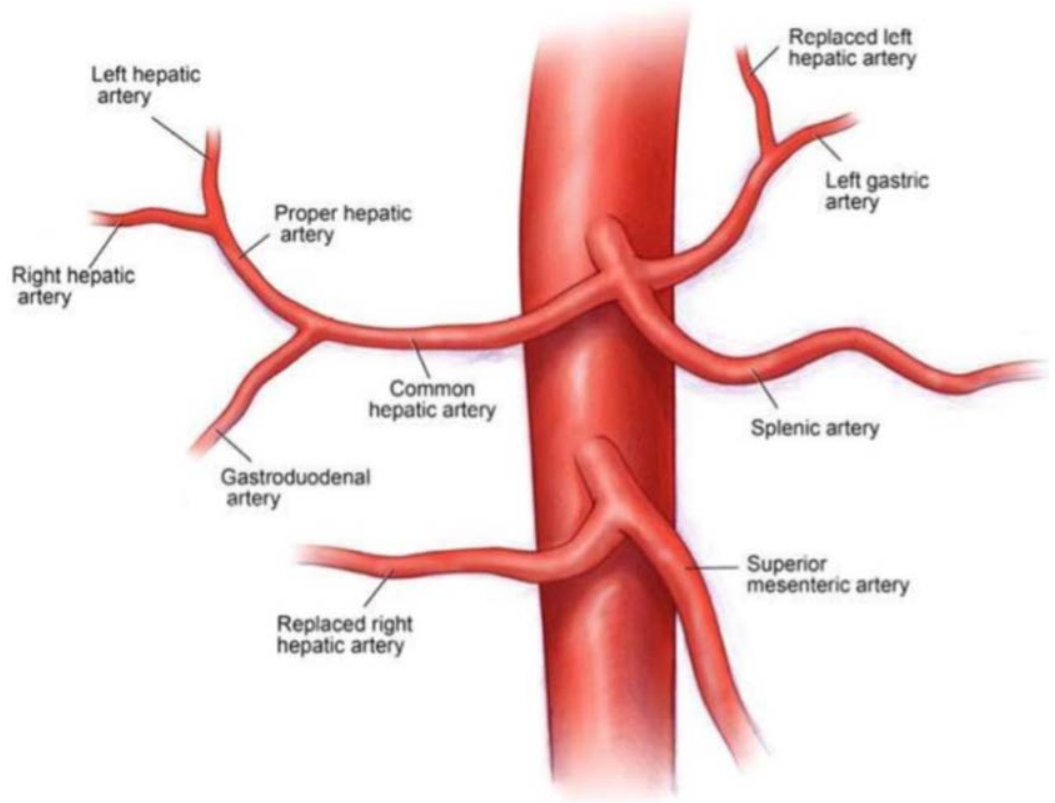

FIGURE 1. Commonly encountered aberrant hepatic artery $(\mathrm{HA})$ anatomy. Variant hepatic vessels originate from a source different than the hepatic artery (ie, an accessory left hepatic coming from the left gastric artery). An "accessory" is a variant vessel that runs parallel to the normal left or right hepatic artery, in contrast to a "replaced" vessel, which provides exclusive arterial supply to that particular lobe (ie, a replaced right hepatic artery arising from the superior mesenteric artery supplies exclusively the right hepatic lobe).

A further, often underappreciated, benefit of NMP is the potential to surgically intervene the graft using a medium in which the impact of this intervention can be evaluated mechanically and functionally in real time without jeopardizing patient safety. Although this potential is often mentioned as a possibility of NMP, to date it has not been used in clinical practice.

Hepatic artery integrity is crucial for the success of liver transplantation. Cannulation of the hepatic artery, as is performed during NMP, does have the potential to cause arterial injury, although no such problems have been reported in clinical studies to date. Meanwhile, recent advances in NMP cannulation technology have enabled dual cannulation techniques to be applied clinically allowing grafts with aberrant hepatic artery anatomy to be perfused successfully. This leaves the implanting surgeon to decide on arterial reconstruction technique-whether to perform these anastomoses in the recipient or consider the alternative of ex vivo reconstruction during NMP. This early report aims to shed light on the latter option, its safety and feasibility in an era in which machine perfusion is likely to reshape liver transplantation practice.

\section{MATERIALS AND METHODS}

The series described herein are cases from the Consortium for Organ Preservation in Europe (COPE) $)^{3}$ randomized controlled trial comparing NMP with static cold storage. The COPE trial recruited a total of 335 cases for randomization between June of 2014 and March 2016, of which 221 were successfully transplanted. From the total group, Birmingham recruited 117 patients and transplanted 105 , being 59 cases $(56 \%)$ randomized to the machine perfusion arm. All the cases included in this case series were performed at a single transplant center. Organs were procured using standard donor after cardiac death or donor after brain death (DBD) retrieval technique, and all grafts randomized to the NMP arm were preserved from donor hospital to implantation using the OrganOx Metra device. As described in detail in the COPE trial protocol, ${ }^{3}$ approval was obtained from national research ethics committees and medical device regulatory bodies in each trial region, the trial protocol was registered before recruitment (ISRCTN 39731134), and all relevant ethical regulations relating to the conduct of this study were followed at each trial site.

Before initiating NMP at the donor hospital, macroscopic evaluation of the graft including presence of steatosis and vascular abnormalities is recorded by retrieval surgeon. Backtable preparation of the liver is then performed after which the organ is cannulated, connected to the machine, and the perfusion commenced. The liver back table is conducted in the standard fashion, with skeletonization of the inferior vena cava (IVC), hepatic artery (HA) as far as the gastroduodenal artery (GDA), and portal vein to the level of its bifurcation. Meticulous hemostatic technique is crucial during this process as it is not sufficient for the organ to be "blood-tight"-the perfusate is composed of packed red cells, gelofusin, and heparin with no platelets or clotting factors present. Therefore, any bleeding that occurs will persist for the duration of the perfusion. The liver needs to be "water-tight."

Once the back table is complete, the cannulation process begins with closure of the suprahepatic IVC (using either with a vascular stapler or suture). The infrahepatic IVC, HA, and portal vein are then cannulated using appropriately sized, wire-reinforced cannulae secured in place using a heavy vicryl ligature (or equivalent) with additional reinforcement of the IVC cannula using a nylon tape snugger. A silicon tube is secured in the bile duct to enable measurement of bile production (Figure 2).

In the 5 cases presented, aberrant arterial anatomy was encountered, which prevented standard single vessel cannulation. Accordingly, a dual arterial cannulation technique was performed using a Y-piece adaptor to attach 2 cannulae to the arterial line (Figure 2). Once in the recipient center, ex vivo hepatic artery 


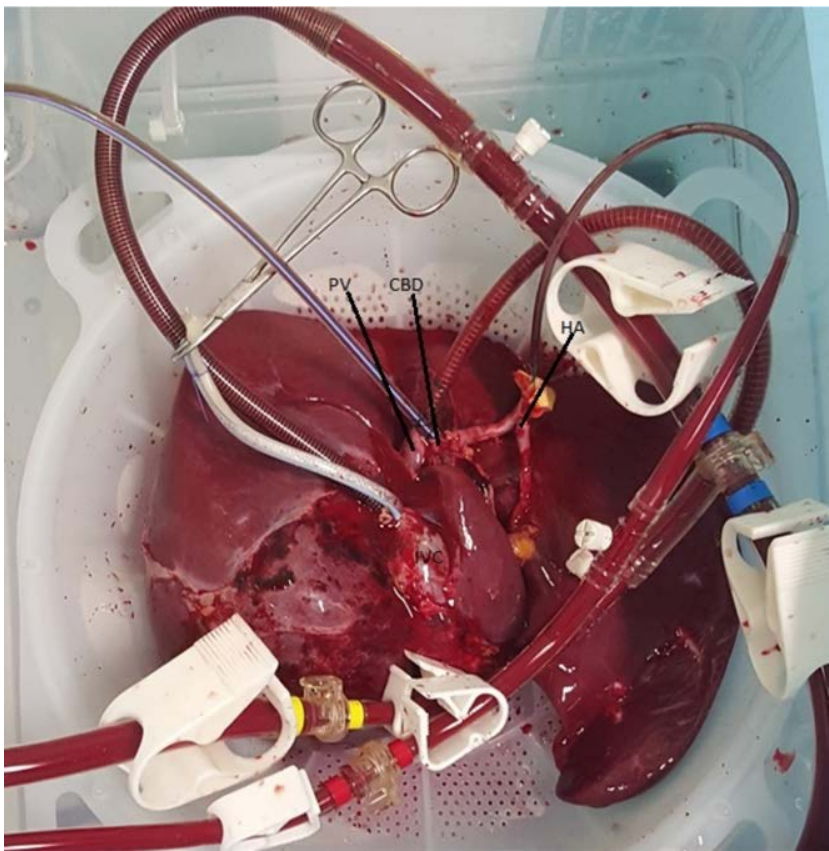

FIGURE 2. A cannulated liver during NMP. CBD, common bile duct; HA, hepatic artery; IVC, inferior vena cava; NMP, normothermic machine perfusion; PV, portal vein.

reconstruction during NMP was performed by the implanting team. A functional evaluation of the reconstruction including anastomotic patency, macroscopic appearance of the graft, and lactate clearance was carried out before transplantation.

Graft implantation was carried out by the participating transplant teams according to standard local protocols. Selection of inflow vessel, magnification, and type of arterial anastomosis was done according to the transplanting surgeon preference. The use of operating microscope was not required in any of the presented cases. In our group, the use of anticoagulation is not standard after an arterial reconstruction, and the use of platelet antiaggregating therapy is common, usually decided by the treating surgeon in a case by case basis.

\section{RESULTS}

We present 5 consecutive cases of ex vivo reconstruction of an aberrant right hepatic artery (RHA) or left hepatic artery (LHA) performed during NMP. In all cases the ex situ reconstruction was performed in the NMP device liver bowl with cannulae in situ during normothermic perfusion using standard reconstructive technique. This was usually an oblique, end-to-end anastomosis of RHA on to splenic or GDA using a continuous, circumferential suture technique with 7-0 prolene. The automated feature of the NMP device allowed flows to be maintained even with significant manipulation of the vessels or cannulae. If flows are compromised this is displayed on the graphical user interface and, if flow is persistently absent, an alarm sounds.

A summary of each case is shown in Table 1. A more detailed description follows below.

\section{Case 1}

A liver from a 72-y-old female DBD donor was accepted for transplant following NMP preservation. During the warm-phase dissection in the donor a replaced RHA was identified arising from the superior mesenteric artery (SMA). The aorta was retrieved intact including celiac artery and SMA (Figure 3A). Following back-table preparation, the aorta was cannulated and perfusion of HA and SMA performed via the aorta (Figure 3B). The organ perfused well achieving HA flows $500 \mathrm{~mL} / \mathrm{min}$ with lactate dropping from $10.1 \mathrm{mmol}$ to 1.3 mmol by $1 \mathrm{~h}$, then falling further to $0.3 \mathrm{mmol}$ by $6 \mathrm{~h}$.

After approximately $15 \mathrm{~h} \mathrm{NMP,} \mathrm{anastomosis} \mathrm{of} \mathrm{the} \mathrm{RHA}$ to the GDA was performed. On completion of this reconstruction HA flow remained at $500 \mathrm{~mL} / \mathrm{min}$ with no change in lactate.

The organ was transplanted successfully into a recipient affected by primary biliary cirrhosis. The time from end of NMP to portal reperfusion was $58 \mathrm{~min}$. HA reperfusion occurred 28 min later. The recipient remains well at $2 \mathrm{y}$.

\section{Case 2}

The liver from a 73-y-old female, donor after cardiac death donor with a previous diagnosis of nonalcoholic steato hepatitis on United States was accepted for transplant following NMP preservation. The functional warm ischemic time was $16 \mathrm{~min}$. The liver weighed approximately $2 \mathrm{~kg}$ with macroscopic appearance of moderate steatosis and some nodularity (Figure 3C). During back-table preparation a replaced RHA was identified arising from the SMA. The HA and SMA were cannulated separately and perfused well, achieving HA flow $400 \mathrm{~mL} / \mathrm{min}$ with lactate falling from $17.6 \mathrm{mmol}$ to $6.1 \mathrm{mmol}$ in the first hour before falling further to $0.8 \mathrm{mmol}$.

After approximately $7 \mathrm{~h}$ NMP, anastomosis of the RHA to the splenic artery was performed (Figure 3D). On completion of this reconstruction HA flow remained at $400 \mathrm{~mL} / \mathrm{min}$ with no change in lactate.

The organ was perfused for a total of $8 \mathrm{~h} 45 \mathrm{~min}$ before being transplanted successfully into a recipient with hepatocellular carcinoma. The time from end of NMP to portal reperfusion was 30 min HA reperfusion occurred 36 min later. The recipient remains well at $2 \mathrm{y}$.

\section{Case 3}

The liver from a 41-y-old male, DBD donor was accepted for transplant following NMP preservation. During the warmphase dissection in the donor, a replaced RHA was identified arising from the SMA. The celiac artery and SMA were cannulated separately (Figure 3E) and perfused well achieving flows of $600 \mathrm{~mL} / \mathrm{min}$ with lactate falling from $10.1 \mathrm{mmol}$ to $1.5 \mathrm{mmol}$ and normalization of most physiological parameters by $1 \mathrm{~h}$ (Figure 3F).

After approximately $4 \mathrm{~h} 30 \mathrm{~min}, \mathrm{NMP}$ anastomosis of the RHA to the splenic artery was performed. On completion of this reconstruction HA flow remained at $600 \mathrm{~mL} / \mathrm{min}$ with no change in lactate.

The organ was transplanted successfully into a recipient with polycystic liver disease. The time from end of NMP to portal reperfusion was 20 min HA reperfusion occurred 40 min later. The recipient remains well at $2 \mathrm{y}$.

\section{Case 4}

The liver from a 57-y-old female, DBD donor was accepted for transplant following NMP preservation. During the warmphase dissection in the donor, an accessory LHA was identified arising from the left gastric artery and an accessory RHA 
arising from the SMA (Figure 3G). Dual cannulation of the celiac and SMA was performed and perfused well achieving flows of $300 \mathrm{~mL} / \mathrm{min}$ with lactate falling to $0.1 \mathrm{mmol}$ by $2 \mathrm{~h}$.

After approximately $17 \mathrm{~h} \mathrm{NMP}$, anastomosis of the RHA to the splenic artery was performed (Figure $3 \mathrm{H}$ ). On completion of this reconstruction HA flow remained at $300 \mathrm{~mL} / \mathrm{min}$ with no change in lactate.

After NMP the graft was transplanted successfully into a recipient with hepatocellular carcinoma. The time from end of NMP to portal reperfusion was $23 \mathrm{~min}$. HA reperfusion occurred 52 min later. The recipient remains well at $2 \mathrm{y}$.

\section{Case 5}

The liver from a 55 -y-old female, DBD donor was accepted for transplant following NMP preservation. The $1.7 \mathrm{~kg}$ liver appeared moderately steatotic with a replaced LHA that was inadvertently divided close to the hilum during the retrieval. This was identified on the back table and a bulldog clip placed across it (Figure 3I) as the vessel length was considered too short for safe cannulation. On commencing NMP lactate dropped from 16 to $1.3 \mathrm{mmol}$ in the first hour of NMP, but there was visible hypoperfusion of the left lobe (Figure 3J left).

On arrival at the transplanting center a 2-stage reconstruction was performed, initially anastomosing the accompanying iliac vessels to the replaced LHA and establishing dual perfusion through the celiac artery and iliac artery.

Once perfusion had been reestablished through the replaced LHA the liver appearance improved (Figure $3 \mathrm{~J}$ right), HA flow increased from 400 to $800 \mathrm{~mL} / \mathrm{min}$ and lactate fell from 1.3 to $0.3 \mathrm{mmol}$. After $7 \mathrm{~h} 43 \mathrm{~min}, \mathrm{NMP}$ the liver was removed from the device and the reconstruction completed in the cold phase with the remaining limb of the iliac vessel anastomosed to the celiac artery. It was then transplanted into a recipient with alcoholic liver disease. The time from end of NMP to portal reperfusion was 73 min HA reperfusion occurred 27 min later. The recipient remains well at $2 \mathrm{y}$.

\section{Outcomes}

All patients survived transplantation, having spent 1-7 d in the intensive care unit and discharged home after 5-15 d. None of the cases developed early allograft dysfunction or any early technical complications including hepatic artery thrombosis. All patients have received at least 2 y follow-up with no evidence of late hepatic artery thrombosis, stenosis, or any other vascular-related complication. Four of the 5 patients underwent magnetic resonance cholangiopancreatography (as part of the trial protocol) at 6 mo with no evidence of ischemic cholangiopathy reported.

\section{DISCUSSION}

The case series described above suggests that minor, yet crucial surgical interventions to the liver graft during ex vivo NMP are feasible, safe, and effective. The preparation and cannulation processes have been identified as the critical steps in achieving a successful normothermic perfusion. Simple interventions such as the repair of minor damage or hemorrhage control are unquestionably safe and often necessary to maintain a successful perfusion. However, until now, the feasibility of more substantial interventions has been uncertain. 


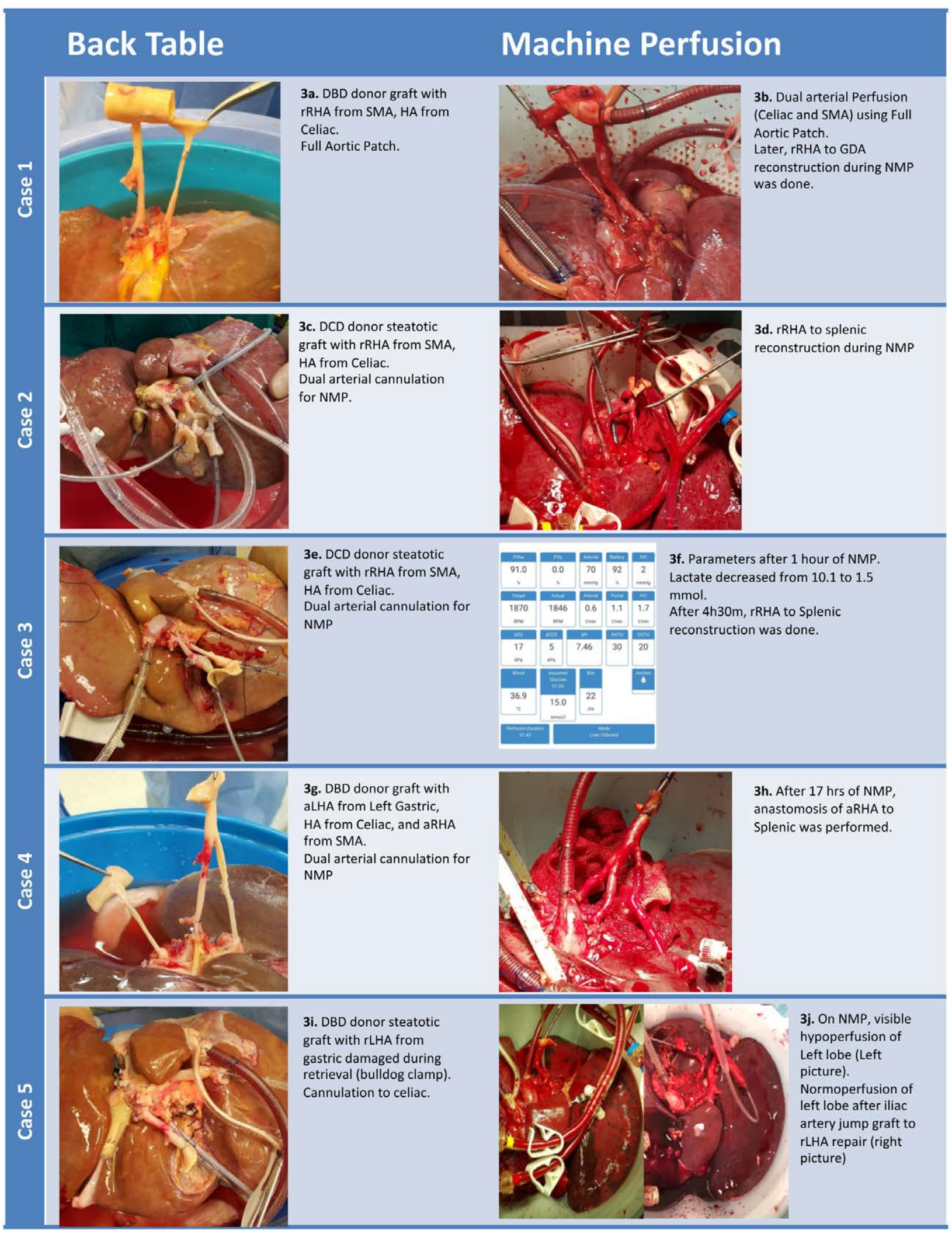

FIGURE 3. Back-table preparation and NMP of 5 arterial reconstruction during NMP cases. aLHA, accessory left hepatic artery; aRHA, accessory right hepatic artery; DBD, donor after brain death; DCD, donor after circulatory death; HA, hepatic artery; NMP, normothermic machine perfusion; NMP, normothermic perfusion; rRHA, replaced left hepatic artery; SMA, superior mesenteric artery.

There are a range of benefits to performing surgical interventions during normothermic perfusion. First, provided the arterial reconstruction is performed using the splenic or GDA, then these branches can be side-clamped while normothermic perfusion continues via the main hepatic artery, supplying the majority of the liver graft without compromising perfusion. 
This prevents the intervention from impacting on the cold ischemia time $^{6}$ or implantation time, ${ }^{7}$ both of which have been shown to be important in preventing later complications.

Second, the necessity of the intervention can be assessed. It is not always apparent if an aberrant hepatic artery is an accessory vessel or a replaced artery supplying an important territory of the liver. As was demonstrated in case 5, this aberrant left hepatic vessel was critical to the perfusion of the left lateral section of the liver. Not only was demarcation of this segment seen when the vessel was clamped but, once normothermic perfusion through this vessel was established, it was accompanied by a further substantial drop in lactate. Such easily measurable changes may be useful in guiding whether it is safe for such a vessel to be sacrificed if, for example, it has been injured during the retrieval process.

Third, performing the reconstruction during NMP allows the mechanical integrity of the reconstruction to be assessed. Any problems can then be corrected without exposing the recipient to the risks of bleeding or extended reconstruction periods once the graft is implanted, should refashioning be required.

We have not observed any complications as a consequence of performing ex vivo arterial reconstruction. In particular, none of the recipients developed biliary complications during their 2-y follow-up or displayed radiological evidence of biliary strictures. This is perhaps surprising given that for the duration of the reconstruction the aberrant vessel's territory is potentially experiencing warm ischemia. However, in this normothermic model, the portal venous supply is also fully oxygenated, which may mitigate the effects of arterial hypoperfusion.

Other groups have already reported performing liver splitting during normothermic perfusion. ${ }^{8}$ This, combined with our experience, suggests that it may be feasible to foresee even major surgical resections during ex vivo normothermic liver perfusion. The logical next step would be to consider performing ex vivo resections of complex hepatic lesions using normothermic perfusion. Such resections are currently performed under conventional cold storage conditions often involving an anhepatic phase of several hours. On autotransplantation of these organs there is a risk or primary nonfunction ${ }^{9}$ or significant hemorrhage, both of which may be avoidable using NMP.

As NMP gains traction in the liver transplant community its wider applications are being explored. Much attention is focused on the development of ex vivo therapeutic interventions such as defatting agents. However, it is apparent that NMP can also be a tool for surgical intervention. Refinements in the design of the NMP liver bowl may be needed if more complex resections are to be considered; at present, the access to the liver is somewhat restricted by the high bowl edges. But the experience to date suggests that such interventions are safe, effective, and may offer advantages over existing ex vivo surgical techniques.

\section{REFERENCES}

1. Michels NA. Blood Supply and Anatomy of the Upper Abdominal Organs With a Descriptive Atlas. Philadelphia, PA: Lipincott; 1955.

2. Molmenti EP, Pinto PA, Klein J, et al. Normal and variant arterial supply of the liver and gallbladder. Pediatr Transplant. 2003;7:80-82. doi:10.1034/j.1399-3046.2003.02046.x

3. Nasralla D, Coussios CC, Mergental H, et al; Consortium for Organ Preservation in Europe. A randomized trial of normothermic preservation in liver transplantation. Nature. 2018;557:50-56. doi:10.1038/ s41586-018-0047-9

4. Watson CJE, Kosmoliaptsis V, Pley C, et al. Observations on the ex situ perfusion of livers for transplantation. Am J Transplant 2018;18:2005-2020. doi:10.1111/ajt.14687

5. Mergental H, Perera MT, Laing RW, et al. Transplantation of declined liver allografts following normothermic ex-situ evaluation. Am J Transplant. 2016;16:3235-3245. doi:10.1111/ajt.13875

6. Porte RJ, Ploeg RJ, Hansen B, et al. Long-term graft survival after liver transplantation in the UW era: late effects of cold ischemia and primary dysfunction. European Multicentre Study Group. Transpl Int. 1998;11(Suppl 1):S164-S167. doi:10.1007/s001470050452

7. Jochmans I, Fieuws S, Tieken I, et al. The impact of implantation time during liver transplantation on outcome: a Eurotransplant cohort study. Transplant Direct. 2018;4:e356. doi:10.1097/ TXD.0000000000000793

8. Brockmann JG, Vogel T, Coussios C, et al. Liver splitting during normothermic organ preservation. Liver Transpl. 2017;23:701-706. doi:10.1002/lt.24693

9. Oldhafer KJ, Lang H, Schlitt HJ, et al. Long-term experience after ex situ liver surgery. Surgery. 2000;127:520-527. doi:10.1067/ msy.2000.105500 